Bundesgesundheitsbl -
In der Diskussion

A. Nassauer · Berlin

\title{
Der Betriebsarzt im Spannungsfeld zwischen Schweigepflicht und Meldepflicht
}

A ner, aber auch in überregionalen Zeitungen zu lesen, daß 13 Patienten, die sich im dortigen Universitätsklinikum einer Herzoperation unterzogen hatten, mit dem Hepatitis-B-Virus (HBV) infiziert worden waren. Alle Patienten hatte der Chefarzt operiert; dies ermittelte das Gesundheitsamt, das sich durch eine Häufung von HBV-Infektionen im Dezember $1998 \mathrm{zu}$ nachgehenden Untersuchungen veranlaßt sah. Daß der Hochschullehrer tatsächlich HBV-Carrier ist und als Infektionsquelle in Betracht kommt, wurde am 1. Februar entdeckt, und das zuständige Wissenschaftsministerium untersagte ihm daraufhin jegliche operative Tätigkeit [1].

In Zeitungsinterviews teilte der Chirurg mit, daß er nie an den klinischen Symptomen einer Hepatitis B (HB) erkrankt war und deshalb auch nicht sagen könne, wann er sich (wahrscheinlich durch einen Patienten) infiziert habe [2]. Eine Hepatitis B-Schutzimpfung hatte er nie erhalten. Außerdem gab er an: „Ich bin in 24 Jahren nicht zu einer einzigen Untersuchung aufgefordert worden." [3].

In anderem Zusammenhang wäre es zweifellos interessant zu klären, welche Vorschriften des Beamtenrechts, des öffentlichen Dienstrechts im weiteren Sinne, des Arbeitsschutzgesetzes, der Biostoff-Verordnung, des Unfallversicherungs-Einordnungsgesetzes (SGB
VII), der Unfallverhütungsvorschriften und berufsgenossenschaftlichen Untersuchungsgrundsätze (z.B. G 42) in diesem Fall nicht beachtet wurden, welche Straftatsbestände zu prüfen sind und inwieweit Klinikum und Chefarzt durch infizierte Patienten zivilrechtlich (auf Schadensersatz und Schmerzensgeld) in Anspruch genommen werden können. Durch die erwähnten Pressemitteilungen ist zum ersten Mal die breite Öffentlichkeit über nosokomiale Virusinfektionen durch einen Chirurgen in Deutschland unterrichtet worden [4].

\section{„In der internationalen Fachliteratur wird über 31 Fälle von HBV- infektiösen Mitarbeitern berichtet, die mindestens 289 Patienten infiziert haben - Übertragungen fanden in erster Linie durch chirurgisch tätige Ärzte statt."}

$\mathrm{Da}$ es sich dabei nicht um ein seltenes Ereignis handelt, haben Hasselhorn und Hofmann belegt, die nach Sichtung der Literatur über 31 Fälle von HBV-infektiösen Mitarbeitern berichten, die mindestens 289 Patienten infiziert hatten. Überträger waren in erster Linie chirurgisch tätige Ärzte; aber auch Infektionen durch Zahnärzte, einen Allgemeinarzt, Kardiotechniker und einen Lungenfunktionslaboranten wurden beschrieben [5].
Was wäre wenn ...

Der geschilderte Sachverhalt hat bei Arbeitsmedizinern umgehend die Frage aufgeworfen, welche Pflichten Personal-, Betriebs- oder D-Ärzten obliegen, wenn durch sie im Rahmen von Einstellungsuntersuchungen, bei Nachuntersuchungen gemäß G 42 oder nach Verletzungen mit Blutkontakt (Arbeitsunfall) der Hepatitis-B-Carrier-Status eines Beschäftigten im Gesundheitswesen festgestellt wird. Verbietet die ärztliche Schweigepflicht in jedem Fall die Weitergabe des Befundes an Dritte? Gibt es arbeitsmedizinische Vorschriften, die sie zur Weitergabe an Arbeitgeber oder Berufsgenossenschaft verpflichten? Enthält das "Seuchenrecht“ Bestimmungen zur Meldung eines Hepatitis B-Carriers an das Gesundheitsamt?

\section{Die ärztliche Schweigepflicht}

Verfassungsrechtliche Grundlage der ärztlichen Schweigepflicht ist Art. 2 Abs. 1 in Verbindung mit Art. 1 Abs. 1 Grundgesetz. Das Bundesverfassungsgericht hat in seiner Entscheidung vom 8.3.1972 ausdrücklich festgestellt: „Wer sich in ärztliche Behandlung begibt, muß und

Dr. Alfred Nassauer

Robert Koch-Institut, Bereich Kreuzberg,

Stresemannstr. 90-102, D-10963 Berlin 
darf erwarten, daß alles, was der Arzt im Rahmen seiner Berufsausübung über seine gesundheitliche Verfassung erfährt, geheim bleibt und nicht zur Kenntnis Unberufener gelangt. Nur so kann zwischen Arzt und Patient jenes Vertrauen entstehen, das zu den Grundvoraussetzungen ärztlichen Wirkens zählt, ...“.

Im Strafrecht hat die ärztliche Schweigepflicht ihren Niederschlag in $\S$ 203 Abs. 1 Nr. 1 StGB gefunden: „Wer unbefugt ein fremdes Geheimnis, namentlich ein zum persönlichen Lebensbereich gehörendes Geheimnis oder ein Betriebs- oder Geschäftsgeheimnis, offenbart, das ihm als Arzt ... anvertraut worden oder sonst bekannt geworden ist, wird mit Freiheitsstrafe bis zu einem Jahr oder mit Geldstrafe bestraft" $[6,7]$.

\section{Schweigepflicht und arbeitsmedizinische Untersuchungen}

Die Aufgaben der Betriebsärzte sind beispielhaft in $\$ 3$ Arbeitssicherheitsgesetz (ASiG) aufgezählt. Der Katalog ist jedoch nicht abschließend. Betriebsärzte unterliegen bezüglich der Gesundheitsdaten der einzelnen Arbeitnehmer prinzipiell der ärztlichen Schweigepflicht wie andere Ärzte auch ( $\$ 8$ Abs. 1 S. 3 ASiG). Hiervon gibt es jedoch aufgabentypische Ausnahmen: Wer sich als Arbeitnehmer bei einer Einstellungsuntersuchung vom Betriebsarzt untersuchen läßt, erklärt damit in aller Regel sein stillschweigendes Einverständnis zur Weitergabe des Untersuchungsergebnisses an den Arbeitgeber. Dieser darf jedoch nur mitteilen, ob gegen die Einstellung des Bewerbers gesundheitliche Bedenken bestehen oder nicht. Einzelheiten zum Befund dürfen nicht offenbart werden.

$\mathrm{Ob}$ eine gleichgelagerte Offenbarungsbefugnis bei allgemeinen arbeitsmedizinischen Vorsorgeuntersuchungen ( $\$ 3$ Abs. 1 Nr. 2 ASiG) im Betrieb angenommen werden kann, ist umstritten. Da der Arbeitnehmer nicht direkt zur Durchführung dieser Vorsorgeuntersuchungen gezwungen werden kann, wird z.T. ein Offenbarungsrecht des Betriebsarztes befürwortet. Dem Arbeitnehmer obliege es, einen ggf. entgegenstehenden Willen zum Ausdruck zu bringen. Dies widerspricht aber dem das Arzt-Patienten-Verhältnis prägende Vertrauensprinzip (BVerfG s.o.). Deshalb ist der Auffassung der Vorzug zu geben, daß bei allgemeinen Vorsorgeuntersuchungen eine Information des Arbeitgebers nur mit dem ausdrücklichen Einverständnis des Arbeitnehmers erfolgen kann. Gerade weil die Untersuchung freiwillig ist, muß der Arbeitnehmer nicht damit rechnen, daß das Ergebnis ohne sein ausdrücklich geäußertes Einverständnis dem Arbeitgeber übermittelt wird. Die bloße Inanspruchnahme einer angebotenen Leistung impliziert nicht den Verzicht auf die Wahrung der eigenen Intimsphäre [8].

\section{"Arbeitsmedizinische Vorsorge- untersuchungen auf $\mathrm{HBV}$ und $\mathrm{HCV}$ sind vorgeschrieben, wenn diese Krankheitserreger am Arbeitsplatz vorkommen können."}

Die Biostoff-Verordnung (BiostVO) ist zum 1. April 1999 in Kraft getreten [9]. Die arbeitsmedizinische Vorsorge ist in $\$ 15$ geregelt. Dort wird festgelegt, daß der Arbeitgeber Beschäftigte vor Aufnahme von Tätigkeiten mit biologischen Arbeitsstoffen nach Anhang IV arbeitsmedizinisch untersuchen und beraten lassen muß. Diese Vorsorgeuntersuchungen sind außerdem in regelmäßigen Abständen zu wiederholen sowie am Ende der Beschäftigung anzubieten. (Da eine Erläuterung zum Rechtsbegriff „in regelmäßigen Abständen“ fehlt, sollten zumindest die Fristen des G 42 eingehalten werden.)

Der Anhang IV beschreibt die verpflichtenden Untersuchungen u.a. für die Humanmedizin und sieht solche auf $\mathrm{HBV}$ und HCV dann vor, wenn diese Krankheitserreger am Arbeitsplatz vorkommen können. Versäumt der Arbeitgeber die beschriebenen Pflichten, handelt er gem. $\$ 18$ Abs. 1 Nr. 11 BiostVO ordnungswidrig; ein Unterlassen kann also sanktioniert werden.

Diese Vorschrift stärkt zweifellos die Position von Personal- und Betriebsärzten, da sie sich hinsichtlich des Un- tersuchungsumfanges jetzt auf eine gesetzliche Regelung berufen können. Allerdings müssen sie auch weiterhin eine Risikoabschätzung zur Festlegung der zu untersuchenden Infektionsparameter vornehmen. Insoweit unterscheiden sich BiostVO und G 42 nicht. Das neue Recht gibt aber einen Mindestkatalog für die Humanmedizin allgemein und speziell erweiterte Untersuchungspflichten von Personal in Kinderabteilungen, Infektionsstationen, Stuhllaboratorien, Tuberkuloseabteilungen und die Pathologie vor. Das Tatbestandsmerkmal „vorkommen können“"verlangt bei Beschäftigten im Gesundheitswesen hinsichtlich HBV und HCV nur die Überlegung, ob bei der jeweiligen beruflichen Tätigkeit Blutkontakte denkbar sind. An eine Risikoabschätzung sind also keine hohen Anforderungen zu stellen.

Auch im zitierten neuen Recht werden - wie in allen anderen relevanten Bestimmungen des medizinischen Arbeitsschutzes - nur Pflichten des Arbeitgebers, jedoch keine Mitwirkungspflichten von Arbeitnehmern oder Beamten beschrieben. Das Erfordernis der Einwilligung zur körperlichen Untersuchung und Blutabnahme ergibt sich regelmäßig nur mittelbar aus den Arbeitsverträgen (als Bedingung mit aufschiebender Wirkung) oder aus dem Beamtenrecht (z.B. $\$ 8$ BBG).

\section{„Die arbeitsmedizinische Untersuchung dient dem Schutz des Beschäftigten, nicht dem des Patienten. Daraus folgt, daß eine Umgehung der Schweigepflicht nicht durch das Arbeitsschutzrecht gedeckt ist."}

Damit wird noch einmal verdeutlicht, daß arbeitsmedizinische Vorsorge allein dem Gesundheitsschutz des Beschäftigten dient. Die körperliche Unversehrtheit von Patienten ist nicht Schutzzweck dieses Rechtsgebietes. Daraus folgert zwingend, daß - eingedenk des geschilderten Beispiels in der Einleitung - eine Umgehung der ärztlichen Schweigepflicht (Weitergabe von Befunden über den HBV-Carrier-Sta- 
tus eines Arztes) grundsätzlich nicht durch das Recht des Arbeitsschutzes gedeckt ist.

Eine Ausnahme bildet $\$ 202$ SGB VII i.V.m. $\S_{5}$ BKVO, wonach ein Arzt im Falle des begründeten Verdachts auf eine Berufskrankheit verpflichtet ist, dies dem Träger der Unfallversicherung oder dem Gewerbearzt anzuzeigen. Darüber hat er den Versicherten zu unterrichten und den Empfänger der Anzeige zu benennen [10].

\section{Schweigepflicht und ärztliches Berufsrecht}

$\$ 9$ der Musterberufsordnung für die deutschen Ärztinnen und Ärzte (MusterBO) [11] beschäftigt sich mit der Schweigepflicht. In Abs. 1 ist der o.g. Grundsatz festgehalten. Abs. 2 beschreibt die hier besonders interessierenden Ausnahmen: „Der Arzt ist zur Offenbarung (von Patientendaten) befugt, soweit er von der Schweigepflicht entbunden worden ist oder soweit die Offenbarung zum Schutz eines höherwertigen Rechtsgutes erforderlich ist. Gesetzliche Aussage- und Anzeigepflichten bleiben unberührt. Soweit gesetzliche Vorschriften die Schweigepflicht des Arztes einschränken, soll der Arzt den Patienten darüber informieren.“ (Dieses Zitat selbst ist nicht geltendes Recht. Aufgrund der Kammer- bzw. Heilberufsgesetze der Länder, beschließen die Delegiertenversammlungen der LÄKn die Berufsordnungen als unmittelbar geltendes Recht für die Kammermitglieder. Im Land Berlin regelt die Schweigepflicht z.B. \$ 3 der Berufsordnung) [12] . Damit ist der gemeinhin als ,ehern“ beschriebene Grundsatz zweifach durchbrochen. Als besonders schwierig dürfte sich eine Abwägung in der Praxis zum Schutz eines höherwertigen Rechtsgutes erweisen.

\section{Meldepflichten gem. Bundes-Seuchengesetz}

$\$_{3}$ BSeuch G beschreibt die Meldepflichtigen und differenziert die Tatbestände nach Erkrankung, Krankheitsverdacht, Tod, Ausscheider und Ausscheidungsverdächtige. Fachliche Einmütigkeit be- steht darin, daß Carrier nicht unter die genannten Tatbestandsmerkmale subsumiert werden können [13]. Allerdings räumt $\$ 7$ Abs. 3 BSeuchG den Ländern das Recht ein, die namentliche Meldepflicht durch Rechtsverordnung zu erweitern. Hiervon haben die Länder Berlin, Brandenburg, Mecklenburg-Vorpommern, Sachsen und Sachsen-Anhalt Gebrauch gemacht. In Thüringen wurde zwischen dem Freistaat, der Kassenärztlichen Vereinigung und der Landesärztekammer vereinbart, daß Meldungen, z.B. eines HBV-Carriers, freiwillig und mit Einverständnis des Betroffenen an das Gesundheitsamt erfolgen sollen (Tabellen 1 und 2).

Damit unterliegt die Meldung eines HBV-Trägers nicht der Schweigepflicht, sondern ein solcher ist zumindest nach dem Recht der neuen Länder und Berlins dem Gesundheitsamt namentlich zu melden. Zwar schweigt das BSeuchG da$\mathrm{zu}$, welche Angaben eine solche Meldung enthalten muß; da sie jedoch das Gesundheitsamt in die Lage versetzen soll, Maßnahmen zur Verhütung ( $\$ 10$ BSeuchG) oder Bekämpfung ( $\$ \$ 34 \mathrm{ff}$. BSeuchG) übertragbarer Krankheiten einzuleiten, sind in den in Deutschland im Umlauf befindlichen Meldeformularen Angaben zu Name, Adresse, Beruf, Laborbefunde und Bemerkungen zu weiteren epidemiologisch relevanten Daten vorgesehen [14]. Dies bedeutet umgekehrt, daß sich für Ärzte aus den alten Ländern aufgrund der $\$ \$ 3 \mathrm{ff}$ BSeuchG keine Meldepflicht ableiten läßt.

\section{Entwurf eines Infektionsschutzgesetzes}

Der Sachstand im März 1999 zum Entwurf für ein Infektionsschutzgesetz sieht ebenfalls keine namentliche Meldepflicht von HBV-Carriern vor. Da aktuell bei der Hepatitis-C-Diagnostik eine Unterscheidung zwischen akuter Infektion und Carrier-Status (Virusnachweis mit ansonsten normalen Laborbefunden) nicht möglich ist, nimmt $\$ 7$ (Meldepflichtige Nachweise von Krankheitserregern) bei HCV-Nachweis in Kauf, daß in Einzelfällen ein Carrier gemeldet wird, denn er schränkt die Meldepflicht nur dann ein, wenn eine chronische Infektion bekannt ist. Abs. 3 der Vorschrift regelt die nichtnamentliche Meldung von Erregernachweisen und führt u.a. HIV auf, so daß z.B. ein solcher Carrier lediglich anonym erfaßt wird.

Gem. $\$ 31$ (Berufliches Tätigkeitsverbot) kann einem bekannten Carrier eine Tätigkeit ganz oder teilweise untersagt werden, soweit dies zum Schutze Dritter vor Infektionen erforderlich ist. $\$ 34$ Abs. 9 sieht vor, daß in Gemeinschaftseinrichtungen, die von einem Carrier besucht werden, Schutzmaßnahmen angeordnet werden können. Aufgrund der Erläuterungen zu den genannten Vorschriften des Gesetzentwurfes wird deutlich, daß seine Autoren die Carrierproblematik abgestuft geregelt wissen möchten. Es bleibt abzuwarten wie insbesondere die neuen Länder auf die zu erwartende Rechtslage reagieren werden. Es ist daran zu erinnern, daß auch die dargelegte Meldepflicht von HBV-Carriern nichts am eingangs geschilderten Sachverhalt geändert hätte (der Carrier-Status des Chirurgen wurde erst Anfang Februar 1999 bekannt).

\section{Schweigepflicht und rechtfertigender Notstand}

Werden Beschäftigte im Gesundheitswesen gem. $\$ 15$ Abs. 1 BiostVO (für den Arbeitgeber verpflichtend) untersucht, ist gem. Anhang IV der VO eine Diagnostik auf HBV und HCV zu veranlassen.

Diesen Tatbestand zugrundelegend befinden sich Betriebsärzte zumindest in den alten Ländern (und in Thüringen) nach der bisherigen Erörterung in einem Dilemma. Weder aufgrund arbeitsmedizinischer Bestimmungen noch nach den Meldetatbeständen des geltenden und des künftigen Infektionsschutzes sind sie befugt, Dritte darüber zu informieren, daß ein Beschäftigter z.B. auf einer Station oder im Operationssaal eines Akutkrankenhauses HBV- oder HCV-Träger ist. Daß zumindest chirurgisch tätige Ärzte dadurch die Gesundheit ihrer Patienten gefährden können, ist belegt [5] und auch für Deutschland seit Anfang dieses Jahres zu bewerten. 


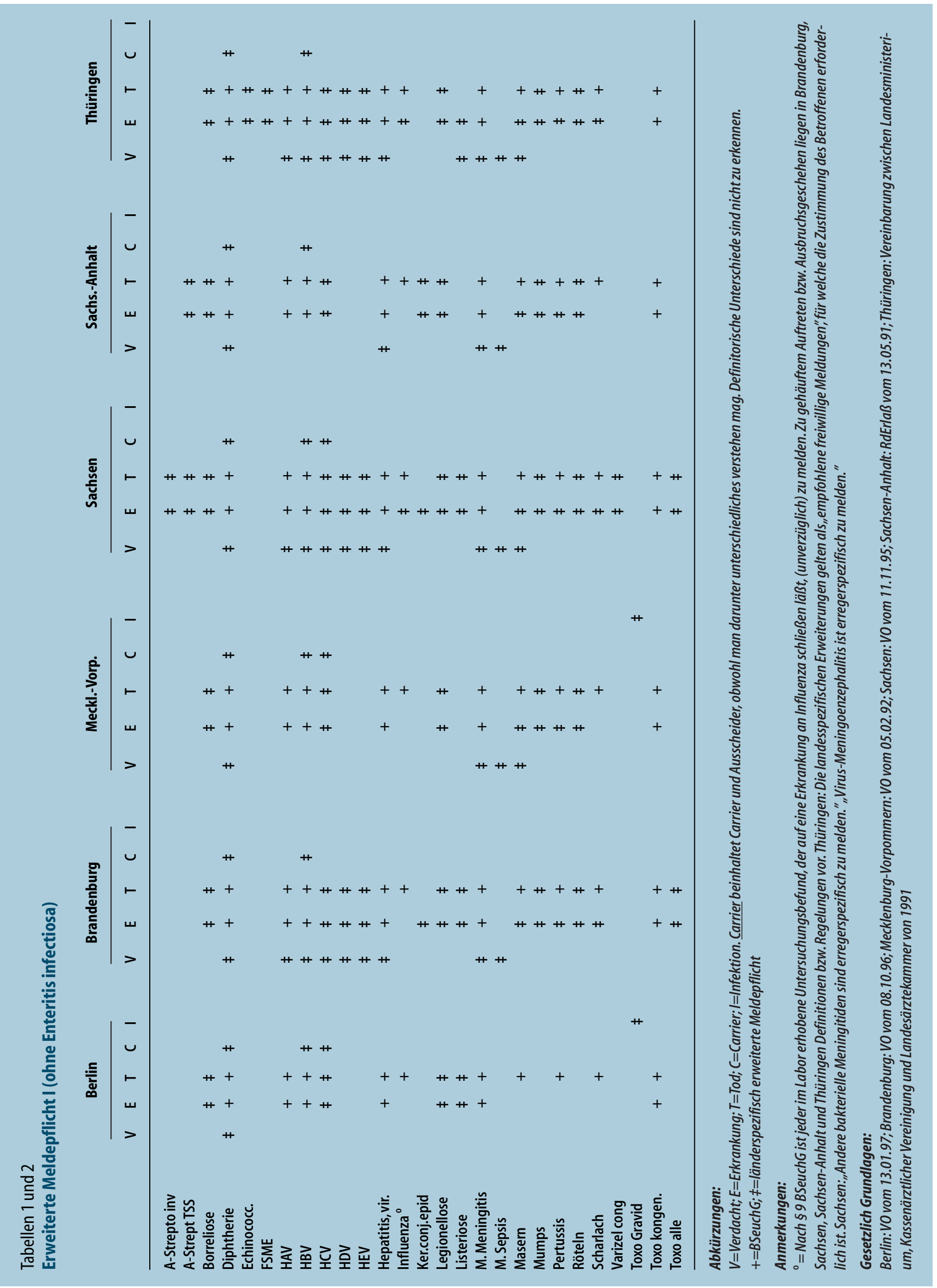


Die Rechtsgrundlage für eine Mitteilung an Dritte (in diesem Fall das Gesundheitsamt), daß z.B. ein chirurgisch tätiger Arzt HBV- oder HCV-Träger ist, bietet der $\S 34$ StGB (Rechtfertigender Notstand). Der Bruch der ärztlichen Schweigepflicht ist danach allerdings nur zulässig, wenn Gefahren nicht anders abwendbar sind und der Bruch der Schweigepflicht ein angemessenes Mittel zur Abwendung potentieller Gefahren für Patienten darstellt.

Eine Lösung bieten $\$ 34$ StGB (Rechtfertigender Notstand) und der schon zitierte $\$ 9$ Abs. 2 der MusterBO für Ärzte, wonach eine Offenbarung zum Schutz eines höherwertigen Rechtsgutes zulässig ist. Zunächst ist die Frage zu beantworten, wem der Befund mitgeteilt werden sollte. Da nach den Arbeitsschutzbestimmungen Beschäftigte freiwillig oder auf Veranlassung des Arbeitgebers zur Untersuchung kommen und der Schutzzweck dieses Rechtsbereiches die Gesundheit des einzelnen Arbeitnehmers ist, sprechen gewichtige Gründe dafür, keine Mitteilung an den Arbeitgeber zu machen. Der Infektionsschutz einzelner Personen wie der Allgemeinheit ist Sinn und Zweck des BSeuchG und des künftigen Infektionsschutzgesetzes. Meldewege und -verfahren sind dort vorgezeichnet, so daß eine Mitteilung an das Gesundheitsamt das sicherlich richtige Vorgehen darstellt.

Die weitaus brisantere Frage ist: Wird durch die Mitteilung, ein Beschäftigter sei HbsAg- und HbeAg-positiv ein „höherwertiges Rechtsgut“ geschützt? Durch eine Meldung werden nämlich seine Grundrechte aus Art. 2 Abs. 1 GG (allgemeines Persönlichkeitsrecht) und Art. 12 Abs. 1 GG (Freiheit der Berufsausübung) tangiert. Dem entgegen steht das Recht der Patienten auf körperliche Unversehrtheit (Art. 2 Abs. 2 GG). Eine Abwägung, welches Grundrecht im Einzelfall ein höherwertiges Rechtsgut darstellt, ist schwierig. Dies ist in der aktuellen Problematik aber auch nicht $\mathrm{zu}$ entscheiden, denn Art. 2 Abs. 1 GG garantiert eine Wahrnehmung dieses Rechtes nur,„,soweit nicht die Rechte an- derer verletzt“" werden. Genau darum geht es aber beim Recht auf körperliche Unversehrtheit der Patienten.

$\$ 34$ StGB gibt eine konkretere Entscheidungshilfe an die Hand. Danach ist der Bruch der Schweigepflicht gerechtfertigt, wenn ein Arzt

in einer gegenwärtigen,

Dicht anders abwendbaren

Defahr

D für Leben, Leib ...

D oder ein anderes Rechtsgut

D eine Tat begeht, (Bruch der Schweigepflicht)

D um die Gefahr ...

Don einem anderen abzuwenden, ...

D Dies gilt jedoch nur, soweit die Tat ein angemessenes Mittel ist.

Gerade der letzte Satz verdient eine genauere Betrachtung. Da die betriebsärztliche Tätigkeit vor allem durch Beratung geprägt ist, muß die Sachlage mit einem Carrier (z.B. einem MRSA-Träger) zunächst vertraulich erörtert werden. Sind Hygienemaßnahmen bekannt, bei deren Einhaltung die Verbreitung bestimmter Krankheitserreger nicht zu befürchten ist, sollten diese dem Betroffenen vermittelt und ihre Einhaltung kontrolliert werden $[15,16]$. Stehen ausreichende bzw. wirksame Schutzmaßnahmen nicht zur Verfügung, sollte der Beschäftigte bewogen werden, einen Wechsel des Arbeitsplatzes mit dem Arbeitgeber unter Hinzuziehung eines sachverständigen Arztes zu erörtern. Erst wenn dieses Verfahren abgelehnt wird oder die Verhandlungen nicht $\mathrm{zu}$ einem befriedigenden Ergebnis führen, ist eine Meldung aufgrund seuchenrechtlicher Vorschriften oder im Rahmen des rechtfertigenden Notstandes sachlich geboten. Konkrete Vorschläge zur Regelung der Beschäftigung von HBV-, HCV- und HIV-infektiösem medizinischen Personal haben Hasselhorn und Hofmann gemacht; in diesem Beitrag werden auch die „Tätigkeiten mit Übertragungsmöglichkeiten “ definiert [17]. Diese Leitlinien sollten zur Beurteilung, ob wirksame Schutzmaßnahmen zur Verfügung stehen (s.o.), herangezogen werden.
Danksagung. Die Tabellen im Anhang hat Herr Dr. E. Werner vom Fachgebiet 21, Epidemiologisches Datenzentrum am RKI, erarbeitet und mir zur Verfügung gestellt, dafür bedanke ich mich sehr herzlich.

\section{Literatur}

1. Die Welt, 9.3.99

2. Bild Zeitung, 8.3.99

3. Focus, $11 / 99$

4. F. Hofmann in Süddeutsche Zeitung, 16.3 .99

5. Hasselhorn HM, Hofmann F (1998) Nosokomiale Hepatitis-B-Virus-, Hepatitis-CVirus- und HIV-Infektionen durch infektiöses medizinische Personal. Gesundheitswesen 60:545-551

6. Sommer A (1998) Die ärztliche Schweigepflicht. Versicherungsmedizin 50:1-2

7. Stein R (1999) Konfliktträchtige Schweigepflicht. Ärzte- und Juristentagung I. Berliner Ärzteblatt, S 25-26

8. Ratzel R, Heinemann N (1999) Ärztliche Schweigepflicht - Sozialgeheimnis Datenschutz. Mikrobiologe 7:170-175

9. Verordnung zur Umsetzung von EG-Richtlinien über den Schutz der Beschäftigten gegen Gefährdung durch biologische Arbeitsstoffe bei der Arbeit. BGBI I 1999, S50-60

10. Blome 0 (1998) Der Arzt im Spannungsfeld zwischen der Schweigepflicht und der Anzeigepflicht von Berufskrankheiten. Pneumologie 52:680-683

11. (Muster-)Berufsordnung für die deutschen Ärztinnen und Ärzte in der Fassung der Beschlüsse des 100. Deutschen Ärztetages in Eisenach. (1997) Deutsches Ärzteblatt 94, C1772-1780

12. Berufsordnung der Ärztekammer Berlin. (1997) Berliner Ärzteblatt, S 23-33

13. Schumacher W, Meyn E (1992) Bundes-Seuchengesetz (Kommentar). 4. Aufl., Deutscher Gemeindeverlag, Köln 1992, Erl.Zu § 6, 23

14. Schumacher W, Meyn E:a.a.o, Erl.zu § 3, 16

15. Für freiwilligen HIV-Test. Gemeinsame Stellungnahme des BMG und der BÄK zum Problem der Übertragung von HIV durch Zahnärzte und Ärzte. Deutsches Ärzteblatt 88 (1991), C1524-1525

16. Hygienische Maßnahmen zur Verhütung der Übertragung von HIV im Krankenhaus (Kommission für Krankenhaushygiene und Infektionsprävention) (1988) Bundesgesundhbl 31,97-98

17. Hasselhorn HM, Hofmann F (1998) Zur Regelung der Beschäftigung von HBV-, HCV- und HIV-infektiösen medizinischen Personals in Deutschland. In: Hofmann F, Jilg W (Hrsg) Nosokomiale Übertragung von HBV, HCV und HIV. Ecomed Verlagsgesellschaft, Landsberg, S 93-95 